OPEN ACCESS

Edited by:

Xiao-Kang Li

National Center for Child Health and

Development (NCCHD), Japan

Reviewed by:

Ling Lu,

Nanjing Medical University, China Masayuki Fujino,

National Institute of Infectious

Diseases (NIID), Japan

*Correspondence:

Shaowei Li

li_shaowei81@hotmail.com

Specialty section:

This article was submitted to Alloimmunity and Transplantation,

a section of the journal

Frontiers in Immunology

Received: 23 February 2020

Accepted: 13 May 2020

Published: 26 June 2020

Citation:

Ye L, He S, Mao X, Zhang Y, Cai Y and

Li S (2020) Effect of Hepatic

Macrophage Polarization and Apoptosis on Liver Ischemia and

Reperfusion Injury During

Liver Transplantation.

Front. Immunol. 11:1193

doi: 10.3389/fimmu.2020.01193

\section{Effect of Hepatic Macrophage Polarization and Apoptosis on Liver Ischemia and Reperfusion Injury During Liver Transplantation}

\author{
Liping Ye ${ }^{1}$, Saiqin $\mathrm{He}^{1,2}$, Xinli Mao ${ }^{1}$, Yu Zhang ${ }^{1}$, Yue Cai ${ }^{1}$ and Shaowei $\mathrm{Li}^{1 *}$ \\ ${ }^{1}$ Department of Gastroenterology, Taizhou Hospital of Zhejiang Province Affiliated to Wenzhou Medical University, Linhai, \\ China, ${ }^{2}$ Endoscopy Center, Taizhou Hospital of Zhejiang Province Affiliated to Wenzhou Medical University, Linhai, China
}

Ischemia-reperfusion (I/R) injury is injury caused by a limited blood supply and subsequent blood supply recovery during liver transplantation. Serious ischemia-reperfusion injury is the main cause of transplant failure. Hepatic $I / R$ is characterized by tissue hypoxia due to a limited blood supply and reperfusion inducing oxidative stress and an immune response. Studies have confirmed that Kupffer cells (KCs), resident macrophages in the liver, play a key role in aseptic inflammation induced by I/R. In liver macrophage polarization, M1 macrophages activated by interferon- $\gamma$ (IFN- $\gamma$ ) and lipopolysaccharide (LPS) exert a pro-inflammatory effect and release a variety of inflammatory cytokines. M2 macrophages activated by IL-4 have an anti-inflammatory response. M1-type KCs are the dominant players in $\mathrm{I} / \mathrm{R}$ as they secrete various pro-inflammatory cytokines that exacerbate the injury and recruit other types of immune cells via the circulation. In contrast, M2-type KCs can ameliorate I/R through unregulated anti-inflammatory factors. A new notion has been proposed that $\mathrm{KC}$ apoptosis may influence I/R in yet another manner as well. Management of KCs is expected to help improve I/R. This review summarizes the effects of hepatic macrophage polarization and apoptosis on liver $\mathrm{l} / \mathrm{R}$.

Keywords: liver transplantation, kupffer cells, ischemia reperfusion, apoptosis, polarization

\section{INTRODUCTION}

Advances in surgical procedures and the application of immunosuppressive technologies have made liver transplantation (LT) the optimal treatment for almost all types of end-stage liver disease (1). The current technology has brought the 1-year survival rate of patients receiving LT to more than $80 \%$, but some problems associated with LT must still be addressed.

The most important factor is I/R (2), which includes ischemic liver damage and subsequent reperfusion injury. It is a two-stage pathophysiological process that occurs during liver resection and liver transplantation (3). Hepatic ischemic injury is characterized by ATP and glycogen depletion as well as cellular metabolic stress caused by mitochondrial dysfunction, all of which lead to initial cell death (4). Subsequent reperfusion injury refers to the phenomenon wherein the liver sustains severe damage after the blood flow and reoxygenation are restored. During this process, metabolic disorders and a large number of reactive oxygen species (ROS) and cytokines or chemokines stimulate various immune cells to produce a severe inflammatory response (5). 
Multiple studies have shown that I/R damage to the liver tissue involves a series of pathological processes (6-8). I/Rrelated macrophage activation and related inflammatory factor explosions are key to graft dysfunction and even the occurrence of primary non-function $(9,10)$.

The liver has several types of innate immune cells, including the inherent macrophages known as Kupffer cells (KCs) (11), dendritic cells (DCs), and natural killer T (NKT) cells. KCs are an important part of the innate immune response and the largest fixed macrophage population in the body, accounting for 40$65 \%$ of the total liver non-parenchymal cells (12). Polarization and apoptosis of KCs have been recognized as important topics concerning hepatic I/R injury.

We herein review the dual role of $\mathrm{KC}$ polarization and apoptosis in I/R.

\section{QUIESCENCE AND ACTIVATION OF KCs IN I/R}

KCs are liver macrophages located in the liver sinusoid and play a key role in the immune response. Under steadystate conditions, KCs have close contact with circulating blood flowing from the portal vein or hepatic artery, which allows them to devour most pathogens. In addition, they are responsible for removing other substances, including cell debris and immune complexes (13). It is now believed that KCs in healthy livers exhibit a "tolerogenic" phenotype that can maintain immune tolerance. However, in a disease state, such as IR, a phenotypic change occurs, which is involved in the immune response $(14,15)$. The activated KCs secrete pro-inflammatory cytokines and induce a subsequent inflammatory response. The activated $\mathrm{KC}$ function is, thus, enhanced, and the cells produce a number of different cytokines and chemokines, such as IL-6, TNF $\alpha$, Nos2, Arg1, and $\operatorname{Mrc1}(16)$.

\section{POLARIZATION OF KCs IN I/R}

KCs are usually polarized to the M1 type during hepatic IR damage (17). It is important that macrophage polarization be understood as a spectrum of transformation. There is no pure M1- or M2-type macrophage population, and these phenotypes undergo transformation according to the stimulation signals they receive (18). M2 macrophages can counteract the pro-inflammatory effects of M1 macrophages during the process of inhibiting pro-inflammatory signaling (19). The pathogenesis of type 2 diabetes (T2D) is chronic hyperinsulinemia caused by systemic and hepatic insulin resistance (IR). Without intervention, pancreatic $\beta$-cell failure will result. IR and T2D are commonly observed in individuals with non-alcoholic fatty liver disease (NAFLD) (20).

Abbreviations: LT, liver transplantation; I/R, ischemia-reperfusion injury; KC, Kupffer cells; ROS, reactive oxygen species; TNF- $\alpha$, tumor necrosis factor $\alpha$; IL, interleukin; DAMPs, Damage-associated molecular patterns; sFGL2, Soluble fibrinogen-like protein 2; DC, dendritic cells.
Hyperglycemia reduces the secretion of IL-10 by inducing the reduction of Arg1 and Mrcl expression and the activation of the STAT3 and STAT6 signaling pathways to inhibit M2like KC polarization. It was demonstrated that hyperglycemia induces high inflammatory activation of KCs during liver I/R. Therefore, the hyperglycemia-induced overexpression of C/EBP homologous protein (CHOP) inhibits the polarization of M2like KCs secreted IL-10, leading to inflammatory activation of KC during liver I/R (21).

PPAR- $\gamma$ exerts a protective effect by inducing $\mathrm{KCs}$ to polarize to M2-type macrophages (22). Sphingosine-1-phosphate (S1P) and sphingosine-1-phosphate receptors (S1PRs) are involved in metabolic and inflammatory diseases. Hyperglycemia exacerbates I/R by promoting M1 polarization and inhibiting M2 polarization, specifically triggering S1P/S1PR3 signaling (23).

Min et al. used myeloid-specific HO-1 gene knockout (mHO-1-KO) and transgenic (mHO-1-Tg) mice to delete or overexpress $\mathrm{HO}-1$, verifying that myeloid $\mathrm{HO}-1$ expression improves liver IR damage by promoting macrophage M2 phenotypic polarization. Interestingly, in human liver transplantation biopsies, subjects with higher HO-1 levels showed a lower expression of M1 markers and higher expression of M2 markers as well as reduced hepatic damage and an improved prognosis (24). Soluble fibrinogen-like protein 2 (sFGL2) promotes the secretion of anti-inflammatory cytokines (IL-10, TGF- $\beta$ ) and the high expression of CD206 and inhibits the activity of STAT1 and NF- $\kappa$ B signaling pathways. sFGL2 improves the prognosis of LT by inducing KC M2 polarization in rat orthotropic liver transplantation (OLT) models (25).

\section{APOPTOSIS OF KCs AND I/R}

A transplanted liver is not only directly affected by $I / R$ but also damaged by apoptosis during transplantation (26). At present, inhibiting and regulating the $\mathrm{KC}$ function has become a hot topic in protecting transplanted livers from I/R. GdCl3 induces $\mathrm{KC}$ apoptosis and reduces IR damage in liver transplantation (27). In the early stages of $I / R$, the activation of KCs and overexpression of inflammatory factors, such as TNF- $\alpha$, are the main causes of graft dysfunction after transplantation (28). KC activation inhibitors are widely used drugs that reduce liver damage in donor animals (29).

KCs exert a protective effect on liver tissue I/R during transplantation (30). Upregulating the expression of IL10 can protect against $\mathrm{I} / \mathrm{R}$ in steatotic liver, and more importantly, KC still has a hepatoprotective effect in steatotic liver (31).

Mesenchymal stem cells (MSCs) have been proposed as promising treatments for certain liver diseases, and studies have found that MSCs also have a protective role in "donated after circulatory death" LT. In a mouse non-heartbeat LT model, the survival rate and cytokine, and chemokine expression of animals with and without MSC infusion were compared. It was found that the protective effect of MSCs on I/R was caused by the 


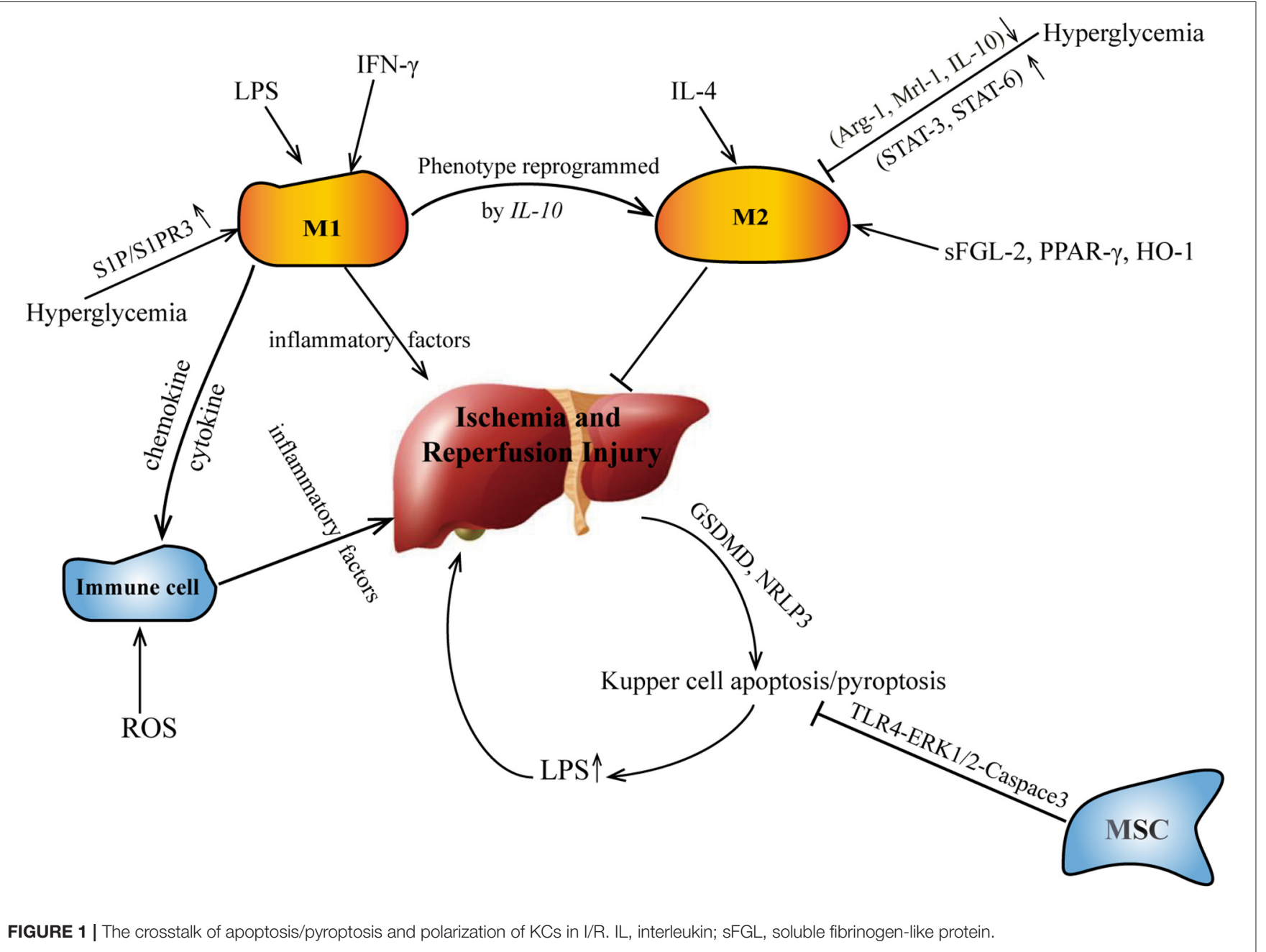

secretion of PGE2, which regulated the TLR4-ERK1/2-caspase3 pathway and inhibited $\mathrm{KC}$ apoptosis (32). KCs, as an important part of the reticuloendothelial system, are responsible for the clearance and detoxification of intestinal Lipopolysaccharide (LPS) (33). Under conditions of KC depletion, LPS is not effectively metabolized in the liver and may continue to cause damage (34). However, indiscriminately reducing $\mathrm{KC}$ activation is not an effective way to reduce $I / R$ in steatosis liver (31).

\section{CROSSTALK OF APOPTOSIS AND POLARIZATION OF KCs IN I/R}

IL-10 is a key anti-inflammatory cytokine produced by immune cells when activated (35). The macrophage phenotype can be reprogrammed from M1 to M2 by upregulating endogenous IL-10 (36). M1 polarization is promoted while M2 polarization is inhibited to specifically aggravate liver I/R (23). In the absence of IL-10 signaling, mTOR can promote the accumulation of damaged mitochondria in macrophages, leading to the dysregulation of NLRP3 inflammatory bodies and production of excess IL-1 $\beta$ (37). In the polarization of primary human macrophages, the expression of apoptosis inhibitor (IAP) protein is different in macrophages with different polarizations. NLR family apoptosis inhibitory protein (NAIP) is highly expressed in M2 macrophages, and cellular IAP 1 (cIAP1) and cIAP2 show opposite expression patterns in M1/M2 polarized macrophages with cIAP1 expressed in M2 and cIAP2 preferentially expressed in M1. Interestingly, IAP antagonists can induce the upregulation of NAIP in M2, downregulation of cIAP1 expression in M1 and M2, and high expression of cIAP2 in M1 macrophages (38).

Cell pyrolysis (pyroptosis), also known as cell inflammatory necrosis, is a new type of programmed cell death (39). It is shown that the permeability of the cell membrane changes, resulting in the release of a large amount of cell contents; at the same time, the water outside the cell enters the cell via channels in the cell membrane. This eventually results in the cell lysing to death, triggering a strong inflammation reaction (40). Liver 
I/R may promote the pyroptosis of KCs mediated by GSDMD and NRLP3 (41). M2-type KCs promote M1-type KC apoptosis through an IL-10-mediated arginase-dependent mechanism (42). The crosstalk of apoptosis/pyroptosis and polarization of KCs in I/R are shown on Figure 1.

\section{CONCLUSION AND FUTURE DIRECTIONS}

Because I/R during LT can increase the risk of graft dysfunction, transplant rejection, and organ failure, managing I/R remains a major problem in clinical practice. I/R activates KCs, and these activated KCs can be polarized into two subtypes. M1type KCs play a pro-inflammatory role, and M2-type KCs play an anti-inflammatory role. The effects of $\mathrm{KC}$ apoptosis on $\mathrm{I} / \mathrm{R}$ development are controversial at present. Interestingly, IL-10 plays a role in attenuating liver $\mathrm{I} / \mathrm{R}$, likely through regulating the apoptosis of KCs as well as modifying their polarization. However, the mechanism underlying liver $I / R$ is not yet fully understood, highlighting the importance of continued research and clarifying the role of KCs in all factors involved in I/R. Such an understanding will aid in the development of more accurate

\section{REFERENCES}

1. Russo FP, Ferrarese A, Zanetto A. Recent advances in understanding and managing liver transplantation. F1000Res. (2016) 5:F1000. doi: 10.12688/f1000research.8768.1

2. Cardoso L, Moreira LFP, Pinto MA, Henriques-Pons A, Alves LA. Domino hepatocyte transplantation: a therapeutic alternative for the treatment of acute liver failure. Can J Gastroenterol Hepatol. (2018) 2018:2593745. doi: 10.1155/2018/2593745

3. Jimenez-Castro MB, Cornide-Petronio ME, Gracia-Sancho J, Peralta C. Inflammasome-mediated inflammation in liver ischemia-reperfusion injury. Cells. (2019) 8:1131. doi: 10.3390/cells8101131

4. Kalogeris T, Baines CP, Krenz M, Korthuis RJ. Cell biology of ischemia/reperfusion injury. Int Rev Cell Mol Biol. (2012) 298:229-317. doi: 10.1016/B978-0-12-394309-5.00006-7

5. Granger DN, Kvietys PR. Reperfusion injury and reactive oxygen species: the evolution of a concept. Redox Biol. (2015) 6:524-51. doi: 10.1016/j.redox.2015.08.020

6. Zhang W, Wang M, Xie HY, Zhou L, Meng XQ, Shi J, et al. Role of reactive oxygen species in mediating hepatic ischemia-reperfusion injury and its therapeutic applications in liver transplantation. Transplant Proc. (2007) 39:1332-7. doi: 10.1016/j.transproceed.2006.11.021

7. Kalogeris T, Baines CP, Krenz M, Korthuis RJ. Ischemia/Reperfusion. Compr Physiol. (2016) 7:113-70. doi: 10.1002/cphy.c160006

8. Soares ROS, Losada DM, Jordani MC, Evora P, Castro ESO. Ischemia/reperfusion injury revisited: an overview of the latest pharmacological strategies. Int $J$ Mol Sci. (2019) 20:5034. doi: 10.3390/ijms20205034

9. Bhogal RH, Sutaria R, Afford SC. Hepatic liver ischemia/reperfusion injury: processes in inflammatory networks-a review. Liver Transpl. (2011) 17:95. doi: 10.1002/lt.22205

10. Datta G, Fuller BJ, Davidson BR. Molecular mechanisms of liver ischemia reperfusion injury: insights from transgenic knockout models. World J Gastroenterol. (2013) 19:1683-98. doi: 10.3748/wjg.v19. i11.1683

11. Lee LY, Kaizu T, Toyokawa H, Zhang M, Ross M, Stolz DB, et al. Carbon monoxide induces hypothermia tolerance in Kupffer cells and attenuates liver ischemia/reperfusion injury in rats. Liver Transpl. (2011) 17:145766. doi: 10.1002/lt.22415 and complete treatment strategies for LT. Future research on I/R in LT should be aimed at developing new therapeutic interventions, implementing prognostic biomarkers based on $\mathrm{KCs}$, and designing clinical studies. It will be necessary to identify new technologies based on regulating the polarization and apoptosis of KCs to encourage the macrophage population to develop in a direction that results in beneficial rather than harmful inflammatory responses.

\section{AUTHOR CONTRIBUTIONS}

LY, SH, XM, YZ, YC, and SL contributed to the writing and editing of the manuscript. All authors contributed to the article and approved the submitted version.

\section{FUNDING}

Name of granting agencies: The Major Research Program of Taizhou Enze Medical Center Grant number: 19EZZDA2. The Program of Taizhou Science and Technology Grant number: $1701 \mathrm{KY} 23$.
12. Lee VG, Johnson ML, Baust J, Laubach VE, Watkins SC, Billiar TR. The roles of iNOS in liver ischemia-reperfusion injury. Shock. (2001) 16:35560. doi: 10.1097/00024382-200116050-00006

13. Ramirez-Pedraza M, Fernandez M. Interplay between macrophages and angiogenesis: a double-edged sword in liver disease. Front Immunol. (2019) 10:2882. doi: 10.3389/fimmu.2019.02882

14. Heymann F, Peusquens J, Ludwig-Portugall I, Kohlhepp M, Ergen C, Niemietz $\mathrm{P}$, et al. Liver inflammation abrogates immunological tolerance induced by Kupffer cells. Hepatology. (2015) 62:279-91. doi: 10.1002/hep.27793

15. Byun JS, Yi HS. Hepatic immune microenvironment in alcoholic and nonalcoholic liver disease. Biomed Res Int. (2017) 2017:6862439. doi: 10.1155/2017/6862439

16. Dong B, Zhou Y, Wang W, Scott J, Kim KH, Sun Z, et al. Vitamin D receptor activation in liver macrophages ameliorates hepatic inflammation, steatosis, and insulin resistance in mice. Hepatology. (2019) 71:155974. doi: 10.1002/hep.30937

17. Nagy LE. The role of innate immunity in alcoholic liver disease. Alcohol Res. (2015) 37:237-50.

18. Yang F, Wang S, Liu Y, Zhou Y, Shang L, Feng M, et al. IRE1alpha aggravates ischemia reperfusion injury of fatty liver by regulating phenotypic transformation of kupffer cells. Free Radic Biol Med. (2018) 124:395407. doi: 10.1016/j.freeradbiomed.2018.06.043

19. Sica A, Mantovani A. Macrophage plasticity and polarization: in vivo veritas J Clin Invest. (2012) 122:787-95. doi: 10.1172/JCI59643

20. Ballestri S, Nascimbeni F, Romagnoli D, Baldelli E, Targher G, Lonardo A. Type 2 diabetes in non-alcoholic fatty liver disease and hepatitis $\mathrm{C}$ virus infection-liver: the "musketeer" in the spotlight. Int J Mol Sci. (2016) 17:355. doi: 10.3390/ijms17030355

21. Rao Z, Sun J, Pan X, Chen Z, Sun H, Zhang P, et al. Hyperglycemia aggravates hepatic ischemia and reperfusion injury by inhibiting liver-resident macrophage M2 polarization via C/EBP homologous protein-mediated endoplasmic reticulum stress. Front Immunol. (2017) 8:1299. doi: 10.3389/fimmu.2017.01299

22. Linares I, Farrokhi K, Echeverri J, Kaths JM, Kollmann D, Hamar M, et al. PPAR-gamma activation is associated with reduced liver ischemia-reperfusion injury and altered tissue-resident macrophages polarization in a mouse model. PLoS ONE. (2018) 13:e0195212. doi: 10.1371/journal.pone.0195212

23. Hu Y, Yang C, Shen G, Yang S, Cheng X, Cheng F, et al. Hyperglycemiatriggered sphingosine-1-phosphate and sphingosine-1-phosphate receptor 3 
signaling worsens liver ischemia/reperfusion injury by regulating M1/M2 polarization. Liver Transpl. (2019) 25:1074-90. doi: 10.1002/lt.25470

24. Zhang M, Nakamura K, Kageyama S, Lawal AO, Gong KW, Bhetraratana $\mathrm{M}$, et al. Myeloid HO-1 modulates macrophage polarization and protects against ischemia-reperfusion injury. JCI Insight. (2018) 3:e120596. doi: 10.1172/jci.insight.120596

25. Pan G, Zhao Z, Tang C, Ding L, Li Z, Zheng D, et al. Soluble fibrinogenlike protein 2 ameliorates acute rejection of liver transplantation in rat via inducing Kupffer cells M2 polarization. Cancer Med. (2018) 7:316877. doi: $10.1002 / \mathrm{cam} 4.1528$

26. Tao T, Chen F, Bo L, Xie Q, Yi W, Zou Y, et al. Ginsenoside Rg1 protects mouse liver against ischemia-reperfusion injury through antiinflammatory and anti-apoptosis properties. J Surg Res. (2014) 191:2318. doi: 10.1016/j.jss.2014.03.067

27. Li JY, Gu X, Zhang WH, Jia S, Zhou Y. GdCl3 abates hepatic ischemiareperfusion injury by inhibiting apoptosis in rats. Hepatobiliary Pancreat Dis Int. (2009) 8:518-23.

28. Gracia-Sancho J, Casillas-Ramirez A, Peralta C. Molecular pathways in protecting the liver from ischaemia/reperfusion injury: a 2015 update. Clin Sci. (2015) 129:345-62. doi: 10.1042/CS20150223

29. Yamanaka K, Houben P, Bruns H, Schultze D, Hatano E, Schemmer P. A systematic review of pharmacological treatment options used to reduce ischemia reperfusion injury in rat liver transplantation. PLoS ONE. (2014) 10:e0122214. doi: 10.1371/journal.pone.0122214

30. Ellett JD, Atkinson C, Evans ZP, Amani Z, Balish E, Schmidt MG, et al. Murine Kupffer cells are protective in total hepatic ischemia/reperfusion injury with bowel congestion through IL-10. J Immunol. (2010) 184:584958. doi: 10.4049/jimmunol.0902024

31. Sutter AG, Palanisamy AP, Ellet JD, Schmidt MG, Schnellmann RG, Chavin KD. Intereukin-10 and Kupffer cells protect steatotic mice livers from ischemia-reperfusion injury. Eur Cytokine Netw. (2014) 25:6976. doi: 10.1684/ecn.2015.0359

32. Tian Y, Wang J, Wang W, Ding Y, Sun Z, Zhang Q, et al. Mesenchymal stem cells improve mouse non-heart-beating liver graft survival by inhibiting Kupffer cell apoptosis via TLR4-ERK1/2-Fas/FasL-caspase3 pathway regulation. Stem Cell Res Ther. (2016) 7:157. doi: 10.1186/s13287-016-0416-y

33. Shao B, Kitchens RL, Munford RS, Rogers TE, Rockey DC, Varley AW. Prolonged hepatomegaly in mice that cannot inactivate bacterial endotoxin. Hepatology. (2011) 54:1051-62. doi: 10.1002/hep.24488

34. Crouser ED, Julian MW, Huff JE, Joshi MS, Bauer JA, Gadd ME, et al. Abnormal permeability of inner and outer mitochondrial membranes contributes independently to mitochondrial dysfunction in the liver during acute endotoxemia. Crit Care Med. (2004) 32:478-88. doi: 10.1097/01.CCM.0000109449.99160.81

35. Ouyang W, Rutz S, Crellin NK, Valdez PA, Hymowitz SG. Regulation and functions of the IL-10 family of cytokines in inflammation and disease. Annu Rev Immunol. (2011) 29:71109. doi: 10.1146/annurev-immunol-031210-101312

36. Yu B, Qin SY, Hu BL, Qin QY, Jiang HX, Luo W. Resveratrol improves CCL4-induced liver fibrosis in mouse by upregulating endogenous IL10 to reprogramme macrophages phenotype from M(LPS) to M(IL4). Biomed Pharmacother. (2019) 117:109110. doi: 10.1016/j.biopha.2019.1 09110

37. Ip WKE, Hoshi N, Shouval DS, Snapper S, Medzhitov R. Antiinflammatory effect of IL-10 mediated by metabolic reprogramming of macrophages. Science. (2017) 356:513-9. doi: 10.1126/science.aa 13535

38. Moron-Calvente V, Romero-Pinedo S, Toribio-Castello S, PlazaDiaz J, Abadia-Molina AC, Rojas-Barros DI, et al. Inhibitor of apoptosis proteins, NAIP, cIAP1 and cIAP2 expression during macrophage differentiation and M1/M2 polarization. PLoS ONE. (2018) 13:e0193643. doi: 10.1371/journal.pone.01 93643

39. Shi J, Gao W, Shao F. Pyroptosis: gasdermin-mediated programmed necrotic cell death. Trends Biochem Sci. (2017) 42:245-54. doi: 10.1016/j.tibs.2016. 10.004

40. Fink SL, Cookson BT. Pillars article: caspase-1-dependent pore formation during pyroptosis leads to osmotic lysis of infected host macrophages. Cell Microbiol. (2006) 8:1812-25. doi: 10.1111/j.1462-5822.2006.0 0751.x

41. Hua S, Ma M, Fei X, Zhang Y, Gong F, Fang M. Glycyrrhizin attenuates hepatic ischemia-reperfusion injury by suppressing HMGB1-dependent GSDMD-mediated kupffer cells pyroptosis. Int Immunopharmacol. (2019) 68:145-55. doi: 10.1016/j.intimp.2019. 01.002

42. Wan J, Benkdane M, Teixeira-Clerc F, Bonnafous S, Louvet A, Lafdil F, et al. M2 Kupffer cells promote M1 Kupffer cell apoptosis: a protective mechanism against alcoholic and nonalcoholic fatty liver disease. Hepatology. (2014) 59:130-42. doi: 10.1002/hep. 26607

Conflict of Interest: The authors declare that the research was conducted in the absence of any commercial or financial relationships that could be construed as a potential conflict of interest.

Copyright $\odot 2020 \mathrm{Ye}, \mathrm{He}, \mathrm{Mao}$, Zhang, Cai and Li. This is an open-access article distributed under the terms of the Creative Commons Attribution License (CC BY). The use, distribution or reproduction in other forums is permitted, provided the original author(s) and the copyright owner(s) are credited and that the original publication in this journal is cited, in accordance with accepted academic practice. No use, distribution or reproduction is permitted which does not comply with these terms. 